\title{
Modulation of Type I Interferon System by African Swine Fever Virus
}

\author{
Elisabetta Razzuoli ${ }^{1, *+\dagger}\left(\mathbb{D}\right.$, Giulia Franzoni ${ }^{2, *}++^{(D}$, Tania Carta ${ }^{2,3}$, Susanna Zinellu ${ }^{2}$, \\ Massimo Amadori ${ }^{4}$, Paola Modesto ${ }^{1}$ and Annalisa Oggiano ${ }^{2}$ \\ 1 Department of Genoa, Istituto Zooprofilattico Sperimentale del Piemonte, Liguria e Valle D'Aosta, \\ 16129 Genova, Italy; paola.modesto@izsto.it \\ 2 Department of Animal Health, Istituto Zooprofilattico Sperimentale della Sardegna, 07100 Sassari, Italy; \\ tcarta@uniss.it (T.C.); susanna.zinellu@izs-sardegna.it (S.Z.); annalisa.oggiano@izs-sardegna.it (A.O.) \\ 3 School of Veterinary Medicine, University of Sassari, 07100 Sassari, Italy \\ 4 Laboratory of Cellular Immunology, Istituto Zooprofilattico della Lombardia e dell'Emilia \\ Romagna (IZSLER), 25124 Brescia, Italy; massimo.amadori@izsler.it \\ * Correspondence: elisabetta.razzuoli@izsto.it (E.R.); giulia.franzoni@izs-sardegna.it (G.F.); \\ Tel.: +39-010542274 (E.R.); +39-0792892358 (G.F.) \\ + These authors have contributed equally to this work.
}

Received: 11 April 2020; Accepted: 7 May 2020; Published: 9 May 2020

\begin{abstract}
African Swine Fever Virus (ASFV) has tropism for macrophages, which seems to play a crucial role in disease pathogenesis and viral dissemination. Previous studies showed that ASFV developed mechanisms to evade type I interferon (IFN) responses. Hence, we analyzed the ability of ASFV strains of diverse virulence to modulate IFN- $\beta$ and IFN- $\alpha$ responses. Porcine monocyte-derived macrophages un-activated (moM $\Phi)$ or activated with IFN- $\alpha(\operatorname{moM} \Phi+$ FN- $\alpha)$ were infected with virulent (22653/14) or attenuated (NH/P68) ASFV strains, and expressions of IFN- $\beta$ and of 17 IFN- $\alpha$ subtypes genes were monitored over time. ASFV strains of diverse virulence induced different panels of IFN genes: infection of moM $\Phi$ with either strains caused statistically significant up-regulation of IFN- $\alpha 3,-\alpha 7 / 11$, whereas only attenuated NH/P68 determined statistically significant up-regulation of IFN- $\alpha 10,-\alpha 12,-\alpha 13,-\alpha 15,-\alpha 17$, and IFN- $\beta$. Infection of activated moM $\Phi$ with either strains resulted in up-regulation of IFN- $\beta$ and many IFN- $\alpha$ subtypes, but statistical significance was found only for IFN- $\alpha 1,-\alpha 10,-\alpha 15,-\alpha 16,-\alpha 17$ in response to NH/P68-infection only. These data revealed differences in type I IFNs expression patterns, with differences between strains of diverse virulence. In addition, virulent 22653/14 ASFV seems to have developed mechanisms to suppress the induction of several type I IFN genes.
\end{abstract}

Keywords: African swine fever; IFN- $\alpha$ subtypes; IFN- $\beta$; monocyte-derived macrophages; INF- $\alpha$ activation

\section{Introduction}

African Swine Fever virus (ASFV) is the etiological agent of African Swine Fever, a contagious viral disease of domestic pigs and wild boar; currently, it is present in many sub-Saharan African countries, Russian Federation, Trans-Caucasus, Eastern and Central Europe, and South East Asia [1,2]. The diffusion of this disease poses a threat to the swine industry worldwide, due to the lack of licensed vaccines or available treatments. Today, stamping out policies and movement restriction are the only measure useful to reduce the spread of the disease, causing important economic losses in the swine industry worldwide [3,4]. The etiological agent is a large, enveloped double-stranded DNA virus, belonging to the Asfaviridae family. ASFV has tropism for cells of the myeloid lineage, especially monocytes and macrophages, which seems to play a crucial role in disease pathogenesis, viral persistence and dissemination [1-3]. In vitro studies on macrophages showed that attenuated 
strains induce enhanced expression of key regulatory cytokines, like IL-12p40, TNF- $\alpha$, and Type I Interferons (IFN- $\beta$ and IFN- $\alpha 1$ ), and chemokines (CCL4, CXCL8, CXCL10) compared to high virulence strains [5-9]. In particular, recent studies demonstrated the ability of virulent ASFV to encode several genes that inhibit Type I IFN responses, i.e.; genes belonging to MGF 360, MGF 505, A276R, A528R, I329L regions [8-10]; deletion of some of these genes results in virulence attenuation [8-11]. Indeed, attenuated ASFV strains, such as NH/P68 and OUTR88/3, which are more sensitive to type I IFN- $\alpha$ [12,13], present loss or truncation of several MGF 360 and 505 genes compared to virulent ASFV strains [14].

IFNs are a family of proteins synthesized and secreted by different cell types. They were named after their capacity to interfere with viral infections [15]; however, they also show immunomodulatory and anti-proliferative activities, revealed in swine, as well [16]. The porcine IFNs system is very complex and among the three groups (I, II, III), porcine type I IFNs family is composed of at least 39 functional genes, including one IFN- $\beta, \alpha \omega,-\varepsilon$, and $-\kappa, 17$ IFN- $\alpha, 11$ IFN- $\delta$, and 7 IFN- $\omega$ subtypes [17]. In pigs, IFN- $\beta$ is encoded by one gene only, whereas IFN- $\alpha$ is a multigene family with 17 functional genes [17]; this gene family shares high identity at both nucleotide (96-99.8\%) and amino acid level $(91.1-100 \%)$ [18]. Despite their high structural homology, porcine IFN- $\alpha$ subtypes exhibit different anti-inflammatory, MHC modulation, and antiviral activities against several pathogens [19-21], thus differences in the production of IFN- $\alpha$ subtypes might influence intensity and duration of an antiviral response. Accordingly, our working hypothesis implied that virulent isolates might have developed mechanisms to suppress selected type I IFN types and subtypes in their target cells; attenuated strains might have lost, at least partially, some of these peculiar mechanisms.

The knowledge of this branch of innate immunity would improve our understanding of the early stage of ASFV pathogenesis and might aid the rational design of ASFV vaccines. In addition, a better understanding of ASFV-driven modulation of type I IFN system might help design antiviral agents or a metaphylactic intervention strategy against this virus. In fact, it has been reported that adenovirus-mediated type I interferon expression delayed appearance and reduced clinical signs in pigs infected with another virus, such as classical swine fever virus (CSFV) [20], and completely protected pigs from foot and mouth disease virus (FMDV) [21]; hence, a similar strategy might be adopted against ASFV, too.

In this conceptual framework, the aim of our study was to investigate the pattern of expression of different type I IFN (IFN- $\beta, 17$ IFN- $\alpha$ subtypes) in response to ASFV infection. We compared an attenuated (NH/P68) and a virulent (22653/14) strains, in order to investigate how the level of attenuation affects the virus ability to induce type I IFN responses.

\section{Results}

\subsection{Evaluation of IFNs Gene Expression on moM $\Phi$}

$\operatorname{moM} \Phi$ were infected with either attenuated NH/P68 or virulent 22653/14 ASFV strains using multiplicity of infection (MOI) 1 ; in our previous work, intracellular levels of late viral proteins p72 and virus infectious particles in culture supernatants were determined at $21 \mathrm{~h}$ pi [13]. We observed that using this MOI $40-60 \%$ of ASFV-infected moM $\Phi$ presented late ASFV protein p72 intracellularly at $21 \mathrm{~h}$ pi [13]. In this work, IFNs gene expression was monitored over time $(3,6,9,12,21 \mathrm{~h} \mathrm{pi})$. Infection with NH/P68 caused a significant increase of IFN- $\beta$ gene expression in moM $\Phi$ at different times pi; in particular, we observed up-regulation at $6(p<0.05), 9(p<0.05), 12(p<0.05), 21(p<0.001)$ hours post-infection with respect to time 0 . On the contrary, the virulent $22653 / 14$ strain did not modulate IFN- $\beta$ gene expression with statistical significance (Figure 1). Concerning the IFN- $\alpha$ subtypes, we observed a statistically significance increase of IFN- $\alpha 3$ and IFN- $\alpha 7 / 11$ gene expression after infection with either NH/P68 or 22653/14 ( $p<0.05$ ) (Figure 1). Increased gene expression was also observed for IFN- $\alpha 9$, but was not statistically significant. NH/P68 caused a broader IFN- $\alpha$ response compared to the virulent $22653 / 14$, with statistically significant induction of IFN- $\alpha 10(p<0.05), \alpha 12(p<0.05),-\alpha 13$ 
$(p<0.05),-\alpha 15(p<0.05),-\alpha 16(p<0.01),-\alpha 17(p<0.05) 24 \mathrm{~h}$ pi (Figure 1$)$. The other genes under study were not significantly $(p>0.05)$ modulated by this virus, although an increase of IFN- $\alpha 5 / 6,-\alpha 8$, $-\alpha 14$ for both strains was observed.
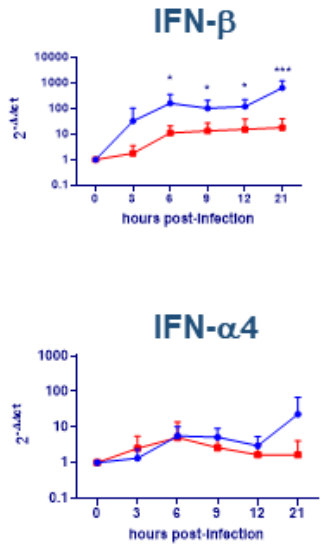

IFN- $\alpha 9$

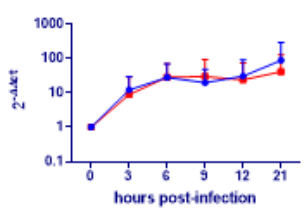

IFN- $\alpha 14$

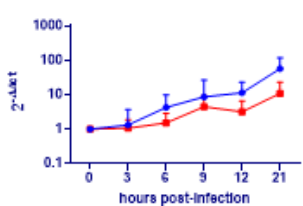

IFN- $\alpha 1$

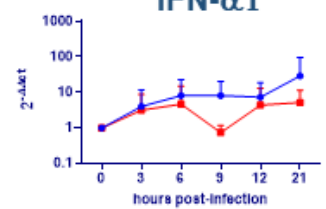

IFN- $\alpha 5 / 6$

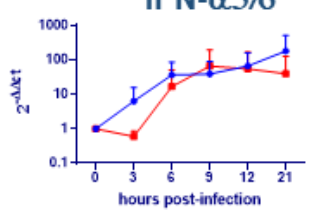

IFN- $\alpha 10$

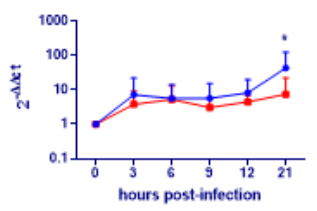

IFN- $\alpha 15$

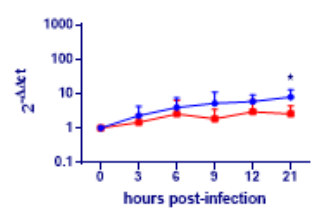

IFN- $\alpha 2$

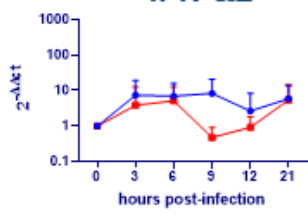

IFN- $\alpha 7 / 11$

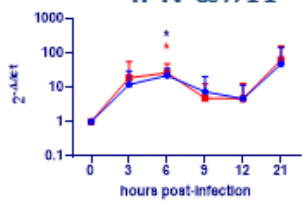

IFN- $\alpha 12$

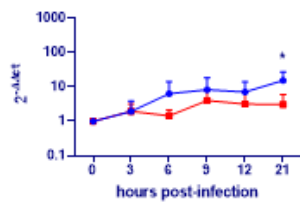

IFN- $\alpha 16$

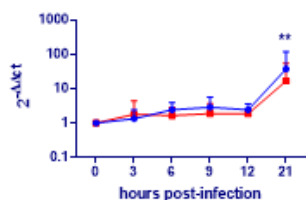

IFN- $\alpha 3$

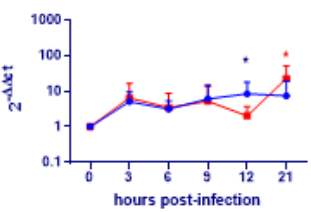

IFN- $\alpha 8$

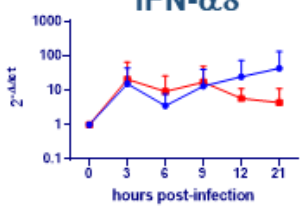

IFN- $\alpha 13$

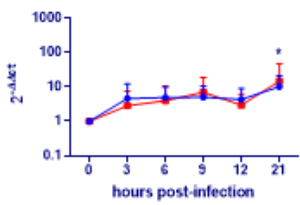

IFN- $\alpha 17$

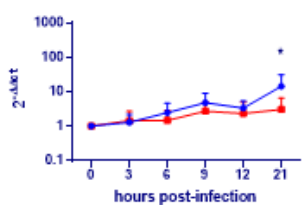

Figure 1. Cytokine gene expression in African Swine Fever Virus (ASFV)-infected macrophages. moM $\Phi$ were infected with the low virulence NH/P68 (blue) or the virulent 22653/14 (red) strains using an MOI 1. At $0,3,6,9,12,21 \mathrm{~h}$ pi, gene expression of IFN- $\beta$ and 17 IFN- $\alpha$ subtypes were determined using qPCR. Data were normalized on the values of un-infected control $(0 \mathrm{~h}$ pi) and expressed as $\Delta \Delta \mathrm{Ct}$, where $\Delta \Delta \mathrm{Ct}=(\Delta \mathrm{Ct}$ observed in un-infected moM $\Phi)-(\Delta \mathrm{Ct}$ observed in ASFV-infected moM $\Phi)$. The mean data + SD from six independent experiments utilizing different animals are shown. Values of ASFV-infected cells were compared to the corresponding mock-infected control using a one-way ANOVA (IFN- $\alpha 12$, IFN- $\alpha 17)$ or a Kruskal-Wallis test (all the others), followed by Dunn's multiple comparison test; ${ }^{* * *} p<0.001,{ }^{* *} p<0.01,{ }^{*} p<0.05$.

\subsection{Evaluation of IFNs Gene Expression on moM $\Phi+I F N-\alpha$}

IFN- $\alpha$ activated moM $\Phi$ were infected with either attenuated NH/P68 or virulent 22653/14 ASFV strains using MOI 1. In our previous work, we observed that using this MOI about $40-50 \%$ of ASFV-infected moM $\Phi$ activated with IFN- $\alpha$ were infected at $21 \mathrm{~h}$ pi [13]. IFNs gene expression was also monitored over time in activated moM $\Phi$. NH/P68 infection of moM $\Phi$ activated with IFN- $\alpha$ caused a significant $(p<0.01)$ increase of IFN- $\beta$ gene expression; in particular, we observed up-regulation, with respect to time 0 , at all time points with the exception of $3 \mathrm{~h}$ post-infection. In addition, the virulent 22653/14 induced moderate IFN- $\beta$ induction, with statistical significance at $9 \mathrm{~h}$ pi $(p<0.05)$ (Figure 2). Regarding IFN- $\alpha$, almost all subtypes were induced, but without statistical significance for IFN- $\alpha 3$ and $-\alpha 7 / 11(p>0.05)$. Both strains induced IFN- $\alpha 2,-\alpha 4,-\alpha 5 / 6,-\alpha 8,-\alpha 9,-\alpha 12,-\alpha 13,-\alpha 14$ induction, but differences were observed between strains: NH/P68 up-regulated $-\alpha 5 / 6,-\alpha 8,-\alpha 12$, $-\alpha 13,-\alpha 14$ at more times pi compared to virulent 22653/14 (Figure 2). NH/P68 only up-regulated gene 
expression of IFN- $\alpha 1$ at $9(p<0.05)$ and $12(p<0.01) h$ pi, IFN- $\alpha 10$ at $9(p<0.05), 12(p<0.01)$ and $21(p<0.05) \mathrm{h}$ pi, and of $-\alpha 15,-\alpha 16,-\alpha 17$ at $12 \mathrm{~h}$ pi $(p<0.05)$ (Figure 2$)$.

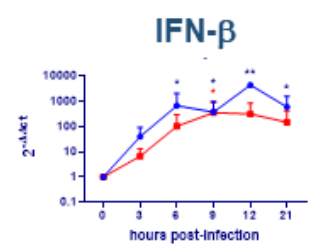

IFN- $\alpha 4$

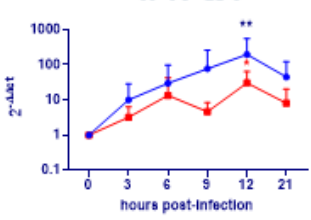

IFN- $\alpha 9$

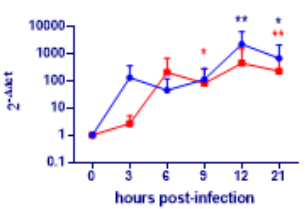

IFN- $\alpha 14$

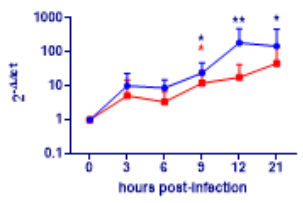

IFN- $\alpha 1$

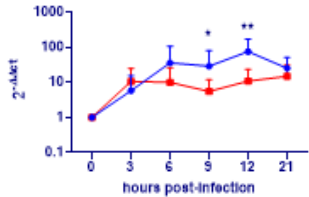

IFN- $\alpha 5 / 6$

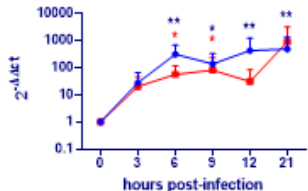

IFN- $\alpha 10$

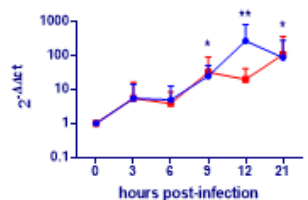

IFN- $\alpha 15$

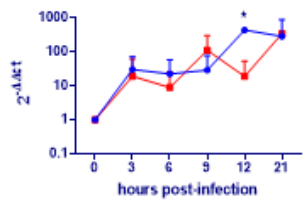

IFN- $\alpha 2$

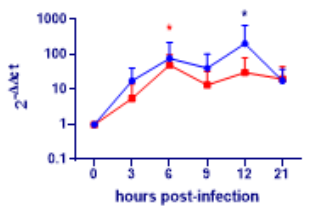

IFN- $\alpha 7 / 11$

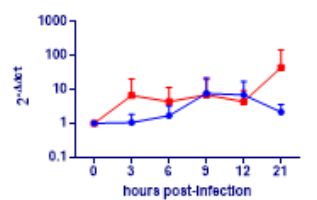

IFN- $\alpha 12$

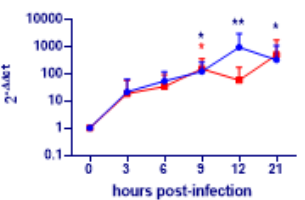

IFN- $\alpha 16$

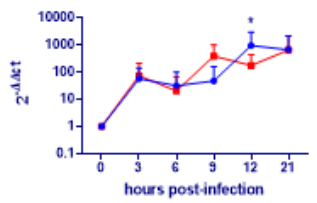

IFN- $\alpha 3$

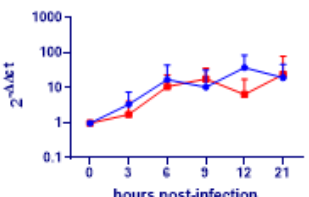

IFN- $\alpha 8$

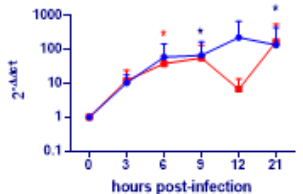

IFN- $\alpha 13$

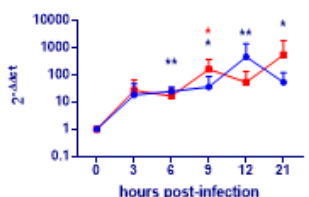

IFN- $\alpha 17$

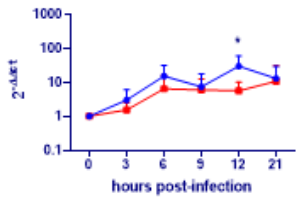

Figure 2. Cytokine gene expression in ASFV-infected IFN- $\alpha$ macrophages. moM $\Phi$ were activated for $24 \mathrm{~h}$ with IFN- $\alpha$ and then infected with the low virulence NH/P68 (blue) or the virulent 22653/14 (red) strains using an MOI 1. At $0,3,6,9,12,21 \mathrm{~h}$ pi, gene expression of IFN- $\beta$ and 17 IFN- $\alpha$ subtypes were determined using qPCR. Data were normalized on the values of un-infected control $(0 \mathrm{~h} \mathrm{pi})$, and expressed as $\Delta \Delta \mathrm{Ct}$, where $\Delta \Delta \mathrm{Ct}=(\Delta \mathrm{Ct}$ observed in un-infected moM $\Phi)-(\Delta \mathrm{Ct}$ observed in ASFV-infected moM $\Phi)$. The mean data + SD from six independent experiments utilizing different animals are shown. Values of ASFV-infected cells were compared to the corresponding mock-infected control using a Kruskal-Wallis test, followed by Dunn's multiple comparison test; ${ }^{* * *} p<0.001$, ** $p<0.01, * p<0.05$.

\subsection{Evaluation of IFNs Release after Virus Infection}

Our ELISA results did not show significant differences $(p<0.05)$ in IFN- $\beta$ and IFN- $\alpha 1$ release after infection with both attenuated or virulent ASFV strains (Figure 3). The same trend was observed for unactivated or IFN- $\alpha$ activated $\operatorname{moM} \Phi$ (Figure 3). Differences between strains mainly concerned IFN- $\beta$ levels in moM $\Phi+$ IFN- $\alpha$ : following 22653/14 infection, secreted levels were lower compared with infection by attenuated NH/P68 strain, although differences were not statistically significant $(p=0.083$, tendency). 
IFN- $\beta$

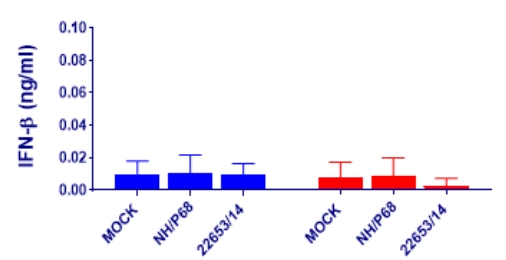

IFN- $\alpha$

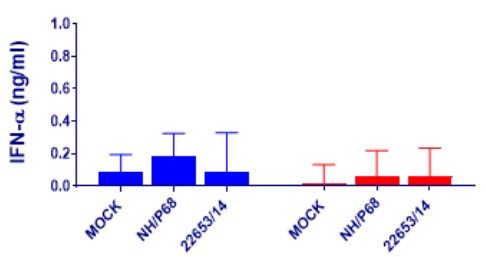

$\operatorname{moM} \Phi$

$\operatorname{moM} \Phi+I F N-\alpha$

Figure 3. IFN- $\beta$ and IFN- $\alpha$ release by macrophage in response to infection with ASFV strains of diverse virulence. moM $\Phi$ were left untreated or activated with IFN- $\alpha .24 \mathrm{~h}$ post-activation, supernatants were removed, then cells were infected with the low virulence NH/P68 or the virulent 22653/14 strains using an MOI 1, alongside mock-infected controls. At $21 \mathrm{~h}$ pi, the amount of IFN- $\beta$, IFN- $\alpha 1$ in culture supernatants were determined using commercial ELISA. The mean data + SD from four (IFN- $\beta$ ) or three (IFN- $\alpha$ ) independent experiments utilizing different animals are shown. Values of ASFV-infected cells were compared to the corresponding mock-infected control using a Kruskal-Wallis test (IFN- $\alpha$ moM $\Phi)$ or a one-way ANOVA followed by Dunn's multiple comparison test; ${ }^{* * *} p<0.001,{ }^{* *} p<0.01$, * $p<0.05$.

\section{Discussion}

Type I IFNs are a heterogeneous group, composed of distinct families (IFN- $\alpha$, IFN- $\beta$, IFN- $\varepsilon$, IFN- $\omega$, IFN- $\kappa$, IFN- $\delta$ and IFN- $\tau$ ), with some of them (like IFN- $\alpha$ ) consisting of different subtypes [17]. In pigs there are 17 IFN- $\alpha$ subtypes, which present different antiviral activities against pseudorabies virus (PRV), classical swine fever virus (CSFV), porcine reproductive and respiratory virus (PRRSV), and vesicular stomatitis virus (VSV) $[17,19,20]$. Therefore, differences in their production by infected cells might influence the quality and the extent of an antiviral response. It can be speculated that virulent AFSV might have developed immune escape mechanisms based on selective inhibition of some IFN- $\alpha$ subtypes.

We observed higher induction of IFN- $\alpha 1$ after infection with the attenuated NH/P68 compared to the virulent $22653 / 14$, with statistical significance in moM $\Phi+$ IFN- $\alpha$, and these findings are in accordance with Gil et al. (2008), where researches observed higher induction of IFN- $\alpha 1$ in macrophages after infection with NH/P68 compared to virulent L60 [6]. IFN- $\alpha 1$ presents strong antiviral activity against PRV [19], PRRSV, and VSV [17], and we recently described that ASFV strain of diverse virulence presented different sensitivity to IFN- $\alpha 1$ antiviral activity: $100 \mathrm{U} / \mathrm{mL}$ of IFN- $\alpha 1$ inhibited NH/P68, but not 22653/14, in $\mathrm{moM} \Phi$, as assessed by reduction of viral levels in culture supernatants [13]. Our results and those of Gil et al. [6] suggest that virulent 22653/14 might have developed mechanisms to suppress induction of this IFN- $\alpha$ subtype, and these are at least partially lost in attenuated NH/P68.

$\mathrm{NH} / \mathrm{P} 68$ infection of moM $\Phi$ resulted in up-regulation of other IFN genes compared to virulent $22653 / 14$, with statistical significance for IFN- $\alpha 10,-\alpha 12,-\alpha 13,-\alpha 15,-\alpha 16,-\alpha 17$. Nevertheless, both strains significantly induced IFN- $\alpha 3$ and IFN- $\alpha 7 / 11$ expression. Interestingly, it was reported that porcine recombinant IFN- $\alpha 3,-\alpha 7,-\alpha 11$ displayed no antiviral activity against PRV [19] and both IFN- $\alpha 7$ and $-\alpha 11$ presented no antiviral activity against PRRSV and VSV [17]. In addition, Sang et al. (2010) observed that PRRSV antiviral activity positively correlated with induction of MxA: all recombinant IFN- $\alpha$ subtypes induced expression of this interferon stimulated gene, with the exception of IFN- $\alpha 7$ and IFN- $\alpha 11$ [17], and the MxA protein can also severely impair ASFV replication [22].

About activated moM $\Phi+$ IFN- $\alpha$, a broader induction of IFN- $\alpha$ subtypes by both strains was observed, but still stronger after infection with NH/P68 compared to 22653/14 ASFV. The virulent Sardinian isolate seems to have developed mechanisms to suppress innate immune responses. NH/P68 only induced gene expression of IFN- $\alpha 1,-\alpha 10,-\alpha 15,-\alpha 16,-\alpha 17$ in activated moM $\Phi$. Interestingly, it was described that IFN- $\alpha 15$ and IFN- $\alpha 16$ downregulated expression of MHC class I [19], and we observed that infection attenuated NH/P68, but not virulent 22653/14, resulted in MHC I downregulation in either moM $\Phi$ or moDC [13-23]. This is likely to provide a crucial advantage to the virulent ASFV 
strain in terms of an outright escape strategy from surveillance of NK cells based on "missing self" [24]. In addition, future studies will hopefully investigate similar mechanisms related to the expression of stress antigens, like MHC class I-related molecules A and B (MICA and MICB) and the family of UL16-binding proteins (ULBP1-6), recognized by NKG2D of NK and NKT cells [24].

Nevertheless, information regarding biological activities of diverse IFN- $\alpha$ subtypes are still limited, and future studies should be performed in order to better understand how different IFNs expression pattern can affect host antiviral response. In vitro experiments, like viral yield reduction assays, will help understand the impact of these molecules on ASFV replication. In addition to that, in vivo studies are badly needed to properly characterize the immunomodulatory activities of these molecules, thus revealing the real extent of their protective effects during ASFV infection.

Concerning IFN- $\beta$, we observed that NH/P68 infection induced higher expression of this gene compared to the virulent 22653/14, in accordance with both Reis et al. (2016) (OURT 88/3 vs. Benin 97/1) and Garcia-Belmonte et al. (2019) (NH/P68 vs. Armenia07) [8,9]. As previously stated, NH/P68 and OUTR88/3 present loss or truncation of several genes within MGF 360 and 505 regions [14], which seem to play a crucial role in suppressing IFN- $\beta$ gene expression [8].

Although our data showed that the attenuated NH/P68 induced higher induction of type I IFN compared to the virulent $22653 / 14$, we could not detect higher production of either IFN- $\alpha 1$ or IFN- $\beta$ from infected $\operatorname{moM} \Phi$, in accordance with a previous study [25]. The authors do not rule out the possible release of minute amounts of Type I IFNs, not revealed by commercial ELISAs and even beneath the usual detection levels of antiviral assays in tissue cultures. The absence of increased type I IFN production by NH/P68-infected macrophages is likely due to post-transcriptional mechanisms, which could involve either mRNA stability, alternative splicing or translation [26]. Nevertheless, lower levels of IFN- $\beta$ were observed in 22653/14-infected compared to mock-infected or NH/P68-infected activated moM $\Phi$. This implies an outright inhibition of IFN- $\beta$ as a result of $22653 / 14$ infection. Differences were not statistically significant, but this statistical tendency was similar to what recently described by Garcia-Belmonte et al. in porcine alveolar macrophages. It was observed that virulent Arm07 ASFV induced IFN- $\beta$ levels lower than those of uninfected controls [9], suggesting that virulent ASFV strains actively suppress the host's immune responses.

Overall, our results highlight that the attenuated NH/P68 induced a stronger type I IFN response compared to virulent $22653 / 14$, with statistically significant induction of IFN- $\beta$ and several IFN- $\alpha$ subtype in $\operatorname{moM} \Phi$. Nevertheless, expression of some IFN- $\alpha$ subtypes $(-\alpha 3$ and $-\alpha 7 / 11)$ in moM $\Phi$ was induced by both strains. Regarding activated moM $\Phi$, both IFN- $\beta$ and diverse IFN- $\alpha$ subtypes were induced by ASFV, but selectively (IFN- $\alpha 1,-\alpha 10,-\alpha 15,-\alpha 16,-\alpha 17)$ or with stronger intensity (IFN- $\beta$, IFN- $\alpha 5 / 6,-\alpha 8,-\alpha 12,-\alpha 13,-\alpha 14$ ) by NH/P68 compared to the virulent Sardinian isolate. Overall, virulent $22653 / 14$ seems to have developed mechanisms to suppress the induction of several type I IFN genes. In addition, we observed that IFN- $\alpha$ subtypes were differently modulated upon infection, highlighting the importance of future studies to better define the biological properties of these subtypes, and to understand how differences in their expression pattern can affect intensity and duration of host antiviral responses. Data generated in this study could contribute to better understand ASFV-driven modulation of type I IFN system, to improve rational design of vaccines or antiviral agents.

\section{Materials and Methods}

\subsection{Animals}

Whole blood was obtained from healthy, 6- to 24-month-old, cross-bred pigs (Sus scrofa domesticus), housed at the Experiment Station of IZS of Sardinia (Sassari, Italy). The local ethical committee approved all procedures performed, in agreement with the Guide of Use of Laboratory Animals issued by the Italian Ministry of Health. The ASFV-negative status was assessed by real time PCR (EDTA blood samples), ELISA (Ingezim PPA Compac ${ }^{\circledR}$, Ingenasa, Madrid, Spain) and Immunoblotting test (serum samples), as described in the Manual of Diagnostic Tests and Vaccines for Terrestrial Animals [27]. 


\subsection{Viruses}

Two ASFV strains of diverse virulence were used in this study: the virulent hemoadsorbing 22653/14 (isolated from a naturally infected pigs during an ASF outbreak in Sardinia in 2014) (Exotic Disease Laboratory ASF Virus Archive, IZS of Sardinia, Sassari, Italy) and the attenuated NH/P68 (kindly provided by the EU ASF Reference Laboratory CISA-INIA, Madrid, Spain). NH/P68 is non-hemoadsorbing and was isolated in 1968 from a chronically infected pig in Portugal [14-28]. Both ASFV strains belong to genotype I $[29,30]$. ASFV strains were propagated in vitro using $25 \mathrm{~cm}^{2}$ flask (Corning, Corning, NY, USA), by inoculation in of sub-confluent monolayers of porcine monocytes/macrophages, as previously described [27-31]. Mock-infected controls were prepared in an identical manner from uninfected monocyte/macrophage cultures. Viral titers were determined by 10 -fold serial dilutions of viral stocks on monocyte/macrophages in 96-well plates, followed by immunofluorescence staining five days post infection [27]. Viral titers were then calculated using the Spearman-Kärber formula.

\subsection{Cells}

Porcine leukocytes were obtained from whole blood and heparin was used as an anticoagulant. Macrophages were generated in Petri dishes using human M-CSF (hM-CSF), as previously described [13-32]. Briefly, leucocytes were cultured at $37{ }^{\circ} \mathrm{C}, 5 \% \mathrm{CO}_{2}$ in Petri dishes in cRPMI (RMPI, $10 \%$ fetal bovine serum (FBS), $100 \mathrm{U} / \mathrm{mL}$ penicillin and $100 \mu \mathrm{g} / \mathrm{mL}$ streptomycin) supplemented with $50 \mathrm{ng} / \mathrm{mL}$ of recombinant hM-CSF (Thermo Fisher Scientific, Waltham, MA, USA) for seven days. Then non-adherent leukocytes were removed, adherent cells were detached by gentle scraping with a pipette, centrifuged at $200 \times g$ for $8 \mathrm{~min}$, and number of viable cells were determined using a Countess Automated Cell Counter (Thermo Fisher Scientific). $5 \times 10^{5}$ live cells/mL were seeded in a 12 well plates (Greiner CELLSTAR, Sigma-Aldrich, Saint Louis, MO, USA) in cRPMI (1.5 mL/well). After seeding, macrophages were cultured at $37^{\circ} \mathrm{C} 5 \% \mathrm{CO}_{2}$ for another $24 \mathrm{~h}$ : they were left untreated (moM $\Phi)$ or stimulated with $100 \mathrm{U} / \mathrm{mL}$ recombinant porcine IFN- $\alpha$ (PBL Assay Science, Piscataway, NJ, USA) $(\operatorname{moM} \Phi+$ IFN- $\alpha)$.

\subsection{ASFV Infection of Macrophage}

$\operatorname{moM} \Phi$ or $\operatorname{moM} \Phi+$ IFN- $\alpha$ were infected with virulent $22653 / 14$ or attenuated NH/P68 using a multiplicity of infection (MOI) of 1 . After $90 \mathrm{~min}$ at $37^{\circ} \mathrm{C} 5 \% \mathrm{CO}_{2}$ (adsorption period), viral inoculum was removed, cells were washed with medium and fresh cRPMI was added to the wells [13]. Cells were incubated at $37{ }^{\circ} \mathrm{C} 5 \% \mathrm{CO}_{2}$ and harvested after 3, 6, 9, 12, $21 \mathrm{~h}$ post-infection (pi), spanning the approximate life cycle of ASFV replication [8,33] At each time points, supernatants were removed, and cells were stored at $-80^{\circ} \mathrm{C}$ until analyzed. Experiments were performed in technical duplicate ( 2 wells each condition) and repeated six times using different blood donor pigs. At $21 \mathrm{~h}$ pi, supernatants were also collected, clarified by centrifugation at $2000 \times g$ for $3 \mathrm{~min}$ and stored at $-80{ }^{\circ} \mathrm{C}$ until analyzed [13].

\subsection{Evaluation of IFNs Gene Expression}

Gene expression of IFN- $\beta$ and 17 different IFN- $\alpha$ subtypes in ASFV-infected moM $\Phi$ at selected time-points $(0,3,6,9,12,21 \mathrm{~h}$ pi) was determined by RT qPCR using primer sets previously described [34,35]. Briefly, in each sample total RNA was extracted using RNeasy Mini Kit (Qiagen, Hilden, Germany) in agreement with the manufacturer's instructions. Then, $100 \mathrm{ng}$ of purified RNA were used as template for cDNA synthesis; EVA Green Real-Time PCR amplification was performed in a CFX96 ${ }^{\mathrm{TM}}$ Real-Time System after the reverse transcription step, as previously described [16]. In each sample, the relative expression of the selected genes was calculated using the formula 2- $\Delta \Delta \mathrm{Ct}$ where:

$$
\Delta \Delta \mathrm{Ct}=\Delta \mathrm{Ct}(\text { mock })-\Delta \mathrm{Ct} \text { (target gene after infection) }
$$


After calculation of 2- $\Delta \Delta \mathrm{Ct}$ and the Kolmogorov-Smirnov test for normality, data sets were checked for statistically significant differences. We performed six experiments using different blood donor pigs.

\subsection{Analysis of Ttype I IFN Levels in Culture Supernatants}

IFN- $\alpha 1$ and IFN- $\beta$ levels in culture supernatants were measured by a Swine IFN- $\alpha$ Do-it-Yourself ELISA kit (King Fisher Biotech, St Paul, MN, USA) or a porcine IFN- $\beta$ ELISA kit (MyBiosource, San Diego, CA, USA), respectively, according to manufacturer's directions. An Epoch microplate reader (BioTek, Winooski, VT, USA) was used to read adsorbance. Experiments were performed in technical duplicate and repeated three (IFN- $\alpha$ ) or four (IFN- $\beta$ ) times using different blood donor pigs.

\subsection{Data Analysis and Statistics}

Experiments were performed in technical duplicate, with three/four (ELISA) or six (qPCR) biological replicates using different pis as the source of $\operatorname{moM} \Phi$. Data are presented as mean with standard deviations (SD). GraphPad Prism v8.01 (GraphPad Software Inc, La Jolla, USA) and Minitab (version of 2019) (Minitab Inc.; Coventry, UK) were used to perform graphical and statistical analysis. All data were checked for normality using the Anderson-Darling test; virus effects on IFNs expression and production were analyzed by the parametric one-way ANOVA or the non-parametric Kruskal-Wallis followed by Dunn's multiple comparison test. The significance threshold was set $p<0.05$. A tendency was declared at $p<0.1$.

Author Contributions: Conceptualization, E.R., G.F.; Methodology, G.F., E.R., T.C., S.Z. and P.M.; Formal Analysis and Data Curation, E.R., G.F., T.C., S.Z., M.A., P.M. and A.O.; Writing-Original Draft Preparation, E.R. and G.F.; Writing -Review \& Editing, M.A. and P.M.; Project Administration, E.R., G.F., P.M. and A.O.; Funding Acquisition, A.O. All authors have read and agreed to the published version of the manuscript.

Funding: This research was funded by the Italian Ministry of Health, grant IZS SA 07/16 and IZSUM PSRC 1/2018.

Acknowledgments: The authors acknowledge Valeria Cimadomo, Ilaria Ferretti (IZS Piemonte, Lombardia, Valle d'Aosta), and Pierpaolo Angioi (IZS della Sardegna) for technical assistance during the study; Paola Nicolussi, Giovannantonio Pilo and all the Animal Services Unit ('Stabulario', IZS della Sardegna, Italy) for the care of animals and provision of blood samples.

Conflicts of Interest: The authors declare no conflict of interest.

\section{References}

1. Sanchez-Cordon, P.J.; Montoya, M.; Reis, A.L.; Dixon, L.K. African swine fever: A re-emerging viral disease threating the global pig industry. Vet. J. 2018, 233, 41-48. [CrossRef] [PubMed]

2. OIE, WAHIS Interface. Available online: http://www.oie.int/wahis_2/public/wahid.php/Diseaseinformation/ Immsummary (accessed on 4 April 2020).

3. Dixon, L.K.; Sun, H.; Roberts, H. African swine fever. Antivir. Res. 2019, 165, 34-41. [CrossRef] [PubMed]

4. Sánchez-Cordón, P.J.; Romero-Trevejo, J.L.; Pedrera, M.; Sánchez-Vizcaíno, J.M.; Bautista, M.J.; Gómez-Villamandos, J.C. Role of hepatic macrophages during the viral haemorrhagic fever induced by African swine fever virus. Histol. Histopathol. 2008, 23, 683-691. [PubMed]

5. Gil, S.; Spagnuolo-Weaver, M.; Canals, A.; Sepúlveda, N.; Oliviera, J.; Aleixo, A.; Allan, G.; Leitão, A.; Martins, C.L. Expression at mRNA level of cytokines and A238L gene in porcine blood-derived macrophages infected in vitro with African swine fever virus (ASFV) isolates of different virulence. Arch. Virol. 2003, 148, 2077-2097. [CrossRef]

6. Gil, S.; Sepúlveda, N.; Albina, E.; Leitão, A.; Martins, C.L. The low-virulent African swine fever virus (ASFV/NH/P68) induces enhanced expression and production of relevant regulatory cytokines (IFNalpha, TNFalpha and IL12p40) on porcine macrophages in comparison to the highly virulent ASFV/L60. Arch. Virol. 2008, 15, 1845-1854. [CrossRef]

7. Fishbourne, E.; Abrams, C.C.; Takamatsu, H.H.; Dixon, L.K. Modulation of chemokine and chemokine receptor expression following infection of porcine macrophages with African swine fever. Vet. Microbiol. 2013, 162, 937-943. [CrossRef] 
8. Reis, A.L.; Abrams, C.C.; Goatley, L.C.; Netherton, C.; Chapman, D.; Sánchez-Cordón, P.J.; Dixon, L.K. Deletion of African swine fever virus interferon inhibitors from the genome of a virulent isolate reduces virulence in domestic pigs and induces a protective response. Vaccine 2016, 34, 4698-4705. [CrossRef]

9. Garcia-Belmonte, R.; Perez-Nunez, D.; Pittau, M.; Richt, J.A.; Revilla, Y. African swine fever virus Armenia/07 virulent strain controls interferon beta production through the cGAS-STING pathway. J. Virol. 2019, 93, e02298-18. [CrossRef]

10. Correira, S.; Ventura, S.; Parkhouse, R.M. Identification and utility of evasion immune defence mechanisms of ASFV. Virus Res. 2013, 173, 87-100. [CrossRef]

11. O'Donnell, V.; Holinka, L.G.; Gladue, D.P.; Sanford, B.; Krug, P.W.; Lu, X.; Arzt, J.; Reese, B.; Carrillo, C.; Risatti, G.R.; et al. African swine fever virus Georgia isolate harbouring deletions of MGF360 and MGF505 genes is attenuated in swine and confers protection against challenge with virulent parental virus. J. Virol. 2015, 89, 6048-6056. [CrossRef]

12. Golding, J.; Goatley, L.; Goodbourn, S.; Dixon, L.; Taylor, G.; Netherton, C. Sensitivity of African swine fever virus to type I interferon is linked to genes within multigene families 360 and 505. Virology 2016, 493, 154-161. [CrossRef] [PubMed]

13. Franzoni, G.; Razzuoli, E.; Dei Giudici, S.; Carta, T.; Galleri, G.; Zinellu, S.; Ledda, M.; Angioi, P.; Modesto, P.; Graham, S.P.; et al. Comparison of macrophage responses to African swine fever viruses reveals that the NH/P68 strain is associated with enhanced sensitivity to type I IFN and and cytokine responses from classically associated macrophages. Pathogens 2020, 9, 209. [CrossRef] [PubMed]

14. Portugal, R.; Coelho, J.; Hoper, D.; Little, N.S.; Smithson, C.; Upton, C.; Martins, C.; Leitao, A.; Keil, G.M. Related strains of African swine fever virus with different virulence: Genome comparison and analysis. J. Gen. Virol. 2015, 96, 408-409. [CrossRef]

15. Isaacs, A.; Lindenmann, J. Virus interference. I. The interferon. Proc. R. Soc. Lond. Ser. B Biol. Sci. 1957, 147, 258-267.

16. Razzuoli, E.; Villa, R.; Ferrari, A.; Amadori, M. A pig tonsil cell culture model for evaluating oral, low-dose IFN- $\alpha$ treatments. Vet. Immunol. Immunopathol. 2014, 160, 244-254. [CrossRef] [PubMed]

17. Sang, Y.; Rowland, R.R.R.; Hesse, R.A.; Blecha, F. Differential expression and activity of the porcine type I interferon family. Physiol. Genom. 2010, 42, 248-258. [CrossRef] [PubMed]

18. Cheng, G.; Chen, W.; Li, Z.; Yan, W.; Zhao, X.; Xie, J.; Liu, M.; Zhang, H.; Zhong, Y.; Zheng, Z. Characterization of the porcine alpha interferon multigene family. Gene 2006, 382, 28-38. [CrossRef]

19. Zanotti, C.; Razzuoli, E.; Crooke, H.; Soule, O.; Pezzoni, G.; Ferraris, M.; Ferrari, A.; Amadori, M. Different biological activities of swine IFN- $\alpha$ subtypes. J. Interferon Cytokine Res. 2015, 35, 990-1002. [CrossRef]

20. Fernandez-Sainz, I.; Ramanathan, P.; O’Donnell, V.; Diaz-San Segundo, F.; Velazquez-Salinas, L.; Sturza, D.F.; Zhu, J.; de Jos Santos, T.; Borca, M.V. Treatment with interferon-alpha delays disease in swine infected with a highly virulent CSFV strain. Virology 2015, 483, 284-290. [CrossRef]

21. Chinsangaram, J.; Moraes, M.P.; Koster, M.; Grubman, M.J. Novel viral disease control strategy: Adenovirus expressing alpha interferon rapidily protects swine from foot-and-mouth disease. J. Virol. 2003, 77, 1621-1625. [CrossRef]

22. Netherton, C.L.; Simpson, J.; Haller, O.; Wileman, T.E.; Takamatsu, H.-H.; Monaghan, P.; Taylor, G. Inhibition of a large double-stranded DNA virus by MxA protein. J. Virol. 2009, 83, 2310-2320. [CrossRef] [PubMed]

23. Franzoni, G.; Graham, S.P.; Sanna, G.; Angioi, P.; Fiori, M.S.; Anfossi, A.; Fiori, M.S.; Anfossi, A.; Amadori, M.; Dei Giudici, S.; et al. Interaction of porcine monocyte-derived dendritic cells with African swine fever viruses of diverse virulence. Vet. Microbiol. 2018, 216, 190-197. [CrossRef] [PubMed]

24. Gonzales, S.; Lopez-Larrea, C.; Lopez-Soto, A. The molecular basis of the immune response to stressed cells and tissues. In The Innate Immune Response to Non-Infectious Stressors, 1st ed.; Amadori, M., Ed.; Academic Press: Cambridge, MA, USA, 2016; pp. 53-79.

25. Portugal, R.; Leitao, A.; Martins, C. Modulation of type I interferon signalling by African swine fever virus (ASFV) of different virulence L60 and NHV in macrophage host cells. Vet. Microbiol. 2018, 216, 132-141. [CrossRef] [PubMed]

26. Khabar, K.S.A.; Young, H.A. Post-transcriptional control of the interferon system. Biochimie 2007, 89, 761-769. [CrossRef]

27. OIE. African Swine Fever. In Manual of Diagnostic Tests and Vaccines for Terrestrial Animals, 7th ed.; Word Organisation for Animal Health: Paris, France, 2012; Volume 2, pp. 1067-1079. 
28. Vigario, J.D.; Terrinha, A.M.; Moura Nunes, J.F. Antigenic relationship among strains of African swine fever virus. Arch. Gesamte Virusforsch. 1974, 45, 272-277. [CrossRef]

29. Gallardo, C.; Mwaengo, D.M.; Macharia, J.M.; Arias, M.; Taracha, E.A.; Soler, A.; Okoth, E.; Martin, E.; Kasiti, J.; Bishop, R.P. Enhanced discrimination of African swine fever virus isolates through nucleotide sequencing of the p54, p72, and pB602L (CVR) genes. Virus Genes 2009, 38, 85-95. [CrossRef]

30. Franzoni, G.; Graham, S.P.; Dei Giudici, S.; Bonelli, P.; Pilo, G.; Anfossi, A.G.; Pittau, M.; Nicolussi, P.S.; Laddomada, A.; Oggiano, A. Characterization of the interaction of African swine fever virus with monocytes and derived macrophage subsets. Vet. Microbiol. 2017, 198, 88-98. [CrossRef]

31. Malmquist, W.A.; Hay, D. Haemadsorbtion and cytopathic effect produced by ASFV in swine bone marrow and buffy coat cultures. Am. J. Vet. Res. 1960, 21, 104-108.

32. Kapentanovic, R.; Fairbairn, L.; Downing, A.; Beraldi, D.; Sester, D.P.; Freeman, T.C.; Tuggle, C.K.; Archibald, A.L.; Hume, D.A. The impact of breed and tissue compartment on the response of pig macrophages to lipopolysaccharide. BMC Genom. 2013, 14, 581.

33. Zhu, J.J.; Ramanathan, P.; Bishop, E.A.; O’Donnell, V.; Gladue, D.P.; Borca, M.V. Mechanisms of African swine fever virus pathogenesis and immune evasion inferred from gene expression changes in infected swine macrophages. PLoS ONE 2019, 14, e0223955. [CrossRef]

34. Razzuoli, E.; Villa, R.; Sossi, E.; Amadori, M. Reverse transcription real-time PCR for detection of porcine interferon alpha and beta genes. Scand. J. Immunol. 2011, 74, 412-418. [CrossRef] [PubMed]

35. Razzuoli, E.; Mignone, G.; Lazzara, F.; Vencia, W.; Ferraris, M.; Masiello, L.; Vivaldi, B.; Ferrari, A.; Bozzetta, E.; Amadori, M. Impact of cadmium exposure on swine enterocytes. Toxicol. Lett. 2018, 287, 92-99. [CrossRef] [PubMed]

(C) 2020 by the authors. Licensee MDPI, Basel, Switzerland. This article is an open access article distributed under the terms and conditions of the Creative Commons Attribution (CC BY) license (http://creativecommons.org/licenses/by/4.0/). 